\title{
POEMS syndrome: a rare cause of adrenal insufficiency in a young male
}

\author{
Joanna Prokop1, João Estorninho', Sara Marote22, Teresa Sabino1, \\ Aida Botelho de Sousa ${ }^{3}$, Eduardo Silva² and Ana Agapito1
}

Departments of ${ }^{1}$ Endocrinology, ${ }^{2}$ Internal Medicine, and ${ }^{3}$ Hemato-Oncology, Centro Hospitalar Universitário Lisboa Central, Lisbon, Portugal
Correspondence should be addressed to J Prokop

Email

Jotka20@gmail.com

\begin{abstract}
Summary
POEMS syndrome (Polyneuropathy, Organomegaly, Endocrinopathy, Monoclonal protein and Skin changes) is a rare multisystemic disease. Clinical presentation is variable, the only mandatory criteria being polyneuropathy and monoclonal gammapathy in association with one major and one minor criterion. Primary adrenal insufficiency is rarely reported. We describe a case of a 33-year-old patient, in whom the presenting symptoms were mandibular mass, chronic sensorymotor peripheral polyneuropathy and adrenal insufficiency. The laboratory evaluation revealed thrombocytosis, severe hyperkalemia with normal renal function, normal protein electrophoresis and negative serum immunofixation for monoclonal protein. Endocrinologic laboratory work-up confirmed Addison's disease and revealed subclinical primary hypothyroidism. Thoracic abdominal CT showed hepatosplenomegaly, multiple sclerotic lesions in thoracic vertebra and ribs. The histopathologic examination of the mandibular mass was nondiagnostic. Bone marrow biopsy revealed plasma cell dyscrasia and confirmed POEMS syndrome. Axillary lymphadenopathy biopsy: Castleman's disease. Gluco-mineralocorticoid substitution and levothyroxine therapy were started with clinical improvement. Autologous hematopoietic cell transplantation (HCT) was planned, cyclophosphamide induction was started. Meanwhile the patient suffered two ischemic strokes which resulted in aphasia and hemiparesis. Cerebral angiography revealed vascular lesions compatible with vasculitis and stenosis of two cerebral arteries. The patient deceased 14 months after the diagnosis. The young age at presentation, multiplicity of manifestations and difficulties in investigation along with the absence of serum monoclonal protein made the diagnosis challenging. We report this case to highlight the need to consider POEMS syndrome in differential diagnosis of peripheral neuropathy in association with endocrine abnormalities even in young patients.
\end{abstract}

\section{Learning points:}

- POEMS syndrome is considered a 'low tumor burden disease' and the monoclonal protein in $15 \%$ of cases is not found by immunofixation.

- Neuropathy is the dominant characteristic of POEMS syndrome and it is peripheral, ascending, symmetric and affecting both sensation and motor function.

- Endocrinopathies are a frequent feature of POEMS syndrome, but the cause is unknown.

- The most common endocrinopathies are hypogonadism, primary hypothyroidism and abnormalities in glucose metabolism.

- There is no standard therapy; however, patients with disseminated bone marrow involvement are treated with chemotherapy with or without HCT. 


\section{Background}

POEMS syndrome is a rare multisystemic disease usually presenting in the fifth and sixth decades (1). The acronym refers to several features of the syndrome: polyneuropathy, organomegaly, endocrinopathy, monoclonal protein and skin changes.

The physiopathology is not well understood; however, it is probably related to an imbalance of proinflammatory cytokines (2). Clinical presentation is variable. The mandatory criteria for diagnosis are polyneuropathy and monoclonal gammapathy in association with at least one major and one minor criterion $(3,4)$.

We report a case of a 33-year-old African male, in whom the presenting symptoms were mandibular mass, chronic sensory-motor peripheral polyneuropathy and adrenal insufficiency.

\section{Case presentation}

A 33-year-old African man presented with a mass on the right side of the neck and was transferred to Portugal from Guinea Bissau in November 2015 for medical care. Two years before, he had complained of a toothache and had a mandibular tumefaction, which was drained in the country of origin. The patient also complained of fatigue, muscular weakness with pain in the upper and lower limbs and unintentional weight loss (about $17 \mathrm{~kg}$ ). He did not have gastrointestinal complaints such as nausea or vomits. He did not notice deepening of skin pigmentation.

After arriving in Portugal, he presented to the Emergency Department with fever, anorexia and general malaise.

Physical examination revealed BP 100/62 mmHg, HR $100 \mathrm{bpm}$, a painful tumefaction of firm consistency in the right side of the face and neck, more pronounced at the malar region, multiple dental cavities, bilateral jugular and axillary lymphadenopathies, asymmetric gynecomastia, diminished breath sounds on pulmonary auscultation and hepatomegaly. The testicular volume was normal, without palpable masses. No skin changes were noted, like hyperpigmentation of palmar lines, or extension surfaces of elbows or knees or mucosa hyperpigmentation. The neurological exam showed motor impairment in the limbs, more pronounced in the legs and hypoesthesia with pain at the upper and lower limbs in a 'stockingglove' pattern. Patellar reflex was absent and the patient had a steppage gait.

\section{Investigation}

Initial work-up at the Emergency Department revealed normochromic, normocytic anemia $(\mathrm{Hb}=10 \mathrm{~g} / \mathrm{dL})$ leukocytosis $(16,000 \mathrm{WBC} / \mu \mathrm{L})$, high platelet count $(705.000 / \mu \mathrm{L})$ and severe hyperkalemia $(8 \mathrm{mEq} / \mathrm{L})$, with normal renal and liver function. Arterial blood gas analysis was normal (Table 1).

An electrocardiogram was performed and showed sinus rhythm with tall peaked $T$ waves in precordial leads. Chest $\mathrm{x}$-ray revealed blunting of the left costophrenic angle.

Maxillofacial, cranial, thoracic and abdominal CT scans were performed. There was swelling and enlargement of the right parotid gland and homolateral masseter, with mandible bone irregularity suggestive of fistule between the third molar and the mandible, with homolateral lymphadenopathies. The chest CT showed condensation with an air bronchogram in the lower lobe of the left lung, discrete subpleural nodularity in the lower lobe of the right lung and focal pleural thickening in the upper lobe of the left lung. There were also signs of centrilobular and parasseptal emphysema in the upper lobes of both lungs and small mediastinal ganglia. The abdominal CT revealed homogeneous hepatosplenomegaly and discreet liquid quantity in abdominal cavity. There were also multiple sclerotic lesions in the thoracic vertebrae, ribs, iliac and left ischium (Fig. 1).

After the initial work-up, the patient was admitted to the Internal Medicine ward and started antibiotic treatment (amoxicillin with clavulanic acid and azithromycin) for a suspected community-acquired pneumonia. The hyperkalemia was initially treated with multiple agents. Afterwards, when the patient maintained only resin therapy, the nadir of potassium concentration was reached on the 7 th day $(5.6 \mathrm{mEq} / \mathrm{L})$.

Laboratory work-up with hepatitis and human immunodeficiency virus (HIV) serology tests as well as an auto-immune study were negative. The serum immune electrophoresis revealed no monoclonal protein, and urinary Bence Jones protein was negative. Serum immunofixation was negative for monoclonal protein. The serum amyloid A protein was elevated when compared with baseline (serum amyloid $A=8.24 \mathrm{mg} / \mathrm{L}$, reference range $<6.4$ ). An interferon-gamma release assay was performed, with indeterminate result.

The patient was submitted to bronchofiberoscopy. Bacilloscopy, cultures of bronchoalveolar lavage fluid (BAL) as well as nucleic acid amplification testing for 
Table 1 Laboratory work-up.

\section{Parameter}

Thyroid-stimulating hormone $(\mu \mathrm{UI} / \mathrm{mL})$

Free T4 (ng/dL)

Follicular stimulating hormone $(\mathrm{mlU} / \mathrm{mL})$

Luteinizing hormone $(\mathrm{mlU} / \mathrm{mL})$

Total testosterone $(\mathrm{ng} / \mathrm{mL})$

Free testosterone $(\mathrm{pg} / \mathrm{mL})$

Prolactin (ng/mL)

Calcium (mg/dL)

Phosphorus (mg/dL)

Parathyroid hormone $(\mathrm{pg} / \mathrm{mL})$

$25 \mathrm{OH}$ vitamin $\mathrm{D}(\mathrm{ng} / \mathrm{mL})$

ACTH at 8:00 h $(\mathrm{pg} / \mathrm{mL})$

Plasma cortisol at 8:00 h $(\mu \mathrm{g} / \mathrm{dL})$

Aldosterone (ng/dL)

Active renin $(\mathrm{uUI} / \mathrm{mL})$

ACTH stimulation test - supine position

Cortisol 0 min $(\mu \mathrm{g} / \mathrm{dL})$

Cortisol $30 \mathrm{~min}(\mu \mathrm{g} / \mathrm{dL})$

Cortisol $60 \mathrm{~min}(\mu \mathrm{g} / \mathrm{dL})$

Aldosterone $0 \mathrm{~min}(\mathrm{ng} / \mathrm{dL})$

Aldosterone $30 \mathrm{~min}$ (ng/dL)

Aldosterone $60 \mathrm{~min}(\mathrm{ng} / \mathrm{dL}$ )

Fasting glucose (mg/dL)

Serum potassium (mEq/L)

Serum sodium (mEq/L)

Urea $(\mathrm{mg} / \mathrm{dL})$

Creatinine $(\mathrm{mg} / \mathrm{dL})$

Urinary sodium (mEq/L)

Urinary potasium (mEq/L)

Urinary chlorine $(\mathrm{mEq} / \mathrm{L})$

Urine anion gap $(\mathrm{mEq} / \mathrm{L})$

Arterial blood gas

$\mathrm{pH}$

$\mathrm{PaO}_{2}(\mathrm{mmHg})$

$\mathrm{PaCO}_{2}(\mathrm{mmHg})$

$\mathrm{HCO}_{3}-(\mathrm{mEq} / \mathrm{L})$

Anion gap ( $\mathrm{mmol} / \mathrm{L})$

\begin{tabular}{c}
\hline Result \\
\hline $6.74 / 6.79^{*}$ \\
$0.76 / 0.77^{*}$ \\
9.29 \\
16.10 \\
2.65 \\
1.83 \\
25.2 \\
7.9 (corrected calcium: 8.3 ) \\
4.8 \\
143.7 \\
11.3 \\
$129 / 109$ \\
8.3 \\
2.3 \\
6.8 \\
\\
6.9 \\
8.00 \\
8.4 \\
1.53 \\
1.59 \\
1.62 \\
73 \\
6.0 \\
132 \\
62 \\
1.17 \\
56 \\
21 \\
124 \\
-47 \\
7.36 \\
96.9 \\
34.9 \\
20 \\
10.6 \\
\\
\\
\\
\\
\\
\\
\\
\\
\end{tabular}

Normal range

$0.35-4.94$

$0.7-1.48$

3.7-19.4

$0.57-12.07$

$1.42-9.23$

$3.8-12.3$

$1.9-25$

8.4-10.2

$2.3-4.7$

14.7-83.1

30-100

$<46$

$1-16$

$2.8-39.9$

$70-100$

3.5-5.1

136-145

19.1-44.1

$0.72-1.25$

7.35-7.45

83-108

$32-48$

$22-26$

$10-14$

* Repeated testing after glucocorticoid replacement therapy.
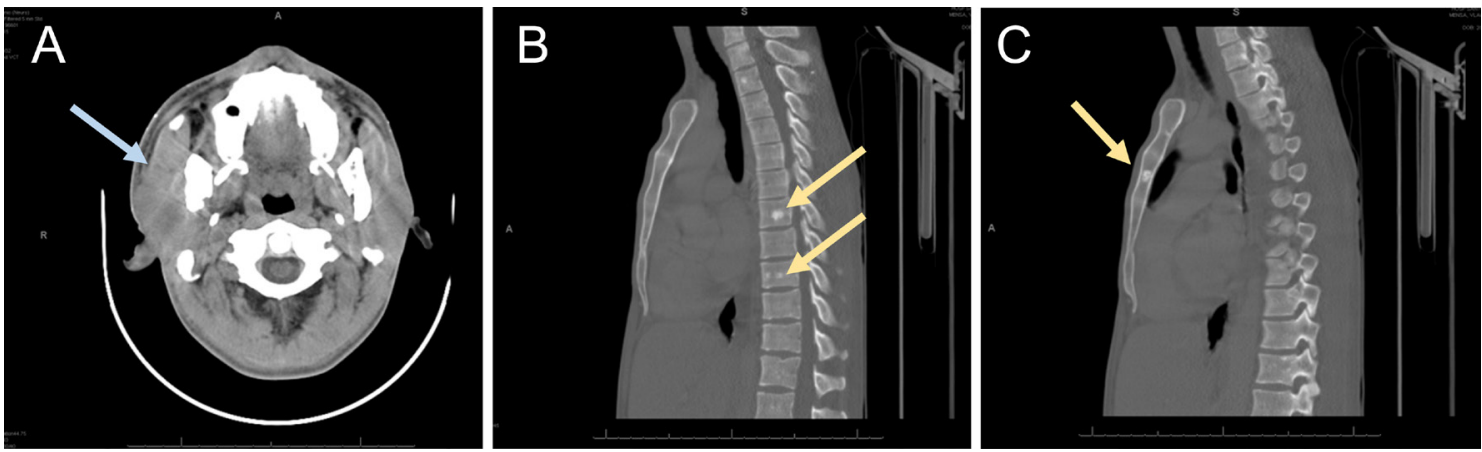

Figure 1

Images from CT scan showing (A) mandibular mass (blue arrow) and (B and C) sclerotic bone lesions (yellow arrows). 
Mycobacterium tuberculosis were performed and were negative.

After clinical improvement, the patient had surgery for removal of the mandibular mass, with nondiagnostic histopathologic examination. Streptococcus mutans and Streptococcus sanguinis were detected by tissue culture (bone and jugal mucosa samples) and osteomyelitis was suspected; hence, the patient was treated with clindamycin and metronidazole.

A subcutaneous abdominal fat biopsy was negative for amyloidosis.

Nerve conduction study was consistent with severe polyneuropathy (sensory and motor) of axonal predominance in the lower limbs and probable predominant demyelization in the upper limbs.

Sural nerve biopsy was performed and was suggestive of demyelinating process and axonal degeneration of undetermined etiology by this technique; there were no lesions suggestive of involvement by inflammatory process, vasculitis or deposition of anomalous substances.

After extensive investigation the patient was discharged in February 2016, under resin therapy, and after a few days he was readmitted to the hospital with worsening of the pain in lower limbs, with edema in the right ankle and worsening of hyperkalemia $(8.1 \mathrm{mEq} / \mathrm{L})$.

During hospitalization, he suffered nosocomial pneumonias and an episode of anasarca with no apparent cause.

After exclusion of a renal cause for hyperkalemia (normal renal function, without any other urinary test abnormalities, as well as absence of metabolic acidosis), the patient was referred to the Endocrinology Department and a laboratory evaluation of the adrenal-pituitary axis was requested, revealing morning cortisol $8.3 \mu \mathrm{g} / \mathrm{dL}$ and ACTH $129 \mathrm{pg} / \mathrm{mL}$ (ACTH on repeated testing $109 \mathrm{pg} / \mathrm{mL}$ ).

The patient was transferred to the Endocrinology Department and a Synacthen test was performed confirming Addison's disease, without any increment of aldosterone level. The DHEAS was not measured. The adrenal antibodies were not tested as the other findings seemed not be related to auto-immune etiology. The CT scan of adrenals was normal. The remaining endocrine evaluation revealed subclinical primary hypothyroidism (confirmed after gluco- and mineralocorticoid replacement, before levothyroxine therapy was started), with thyroid antibodies in the normal range, mild hypocalcemia with high parathyroid hormone (PTH) level and low vitamin $\mathrm{D}$, and an increase of gonadotrophic hormones with total testosterone within the reference range and low free testosterone; however, it was measured by radioimmunoassay (Table 1 ).

The combination of chronic sensory-motor peripheral polyneuropathy, adrenal insufficiency as well as the association of other endocrinopathies, and sclerotic bone lesions prompted suspicion of POEMS syndrome. The patient was referred to the hematologist, and a bone marrow aspiration along with biopsy was performed. The biopsy showed hypercellular marrow (80\%/20\% adipocytes) with trilinear hematopoiesis, maturation and dysplasia of megakaryocytes, mild bone marrow stroma fibrosis and $10 \%$ monoclonal lambda chain plasmocytosis, confirming plasma cell dyscrasia.

An axillary lymph node biopsy was compatible with hyaline vascular variant of Castleman's disease (CD) (Fig. 2).

\section{Differential diagnosis}

The association of peripheral neuropathy, lymphadenopathy, hepatosplenomegaly and gynecomastia raised suspicion of a multisystemic disorder. Once the diagnosis of adrenal insufficiency was made, the presence of osteosclerotic lesions and thrombocytosis led to the hypothesis of POEMS syndrome, even though a monoclonal protein was not detectable in serum on analysis by immunofixation. The combination of peripheral neuropathy and Addison's disease in a young male could also be related to X-linked adrenoleukodystrophy; however, this diagnosis seemed to be less likely due to the presence of other manifestations.

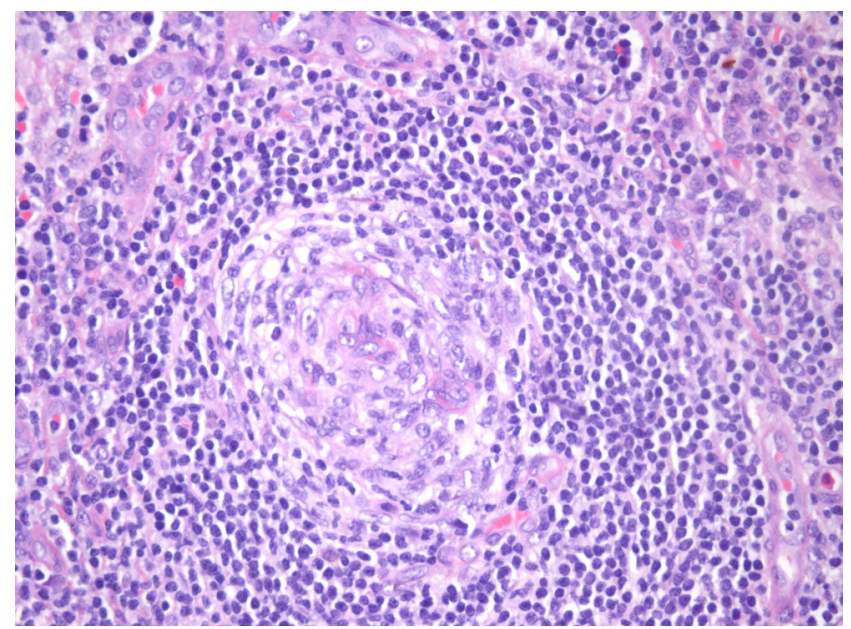

Figure 2

Lymph node biopsy: follicle with small germinative center and expansion of the mantle zone. There are hyalinized vessels and lymphocytes arranged concentrically. 
Table 2 Diagnostic criteria of POEMS syndrome.

\section{Criteria}

Mandatory criteria

1. Polyneuropathy

2. Monoclonal plasma cell proliferative disorder

Major criteria

1. Castleman's disease

2. Sclerotic bone lesions

3. VEGF elevation

Minor criteria

1. Organomegaly (splenomegaly, hepatomegaly or lymphadenopathy)

2. Extravascular volume overload

3. Endocrinopathy

4. Skin changes (hyperpigmentation, hypertrichosis, plethora, acrocyanosis, flushing, white nails)

5. Papilloedema

6. Thrombocytosis or polycythaemia

Other symptoms and signs

Clubbing, weight loss, hyperhidrosis, pulmonary hypertension/restrictive lung Weight loss

disease, thrombotic diatheses, diarrhea, low vit. B12 values Not tested

Absent

Absent

Features seen in our patient

Present, demyelinating

Confirmed by bone marrow biopsy

Demonstrated by lymph node biopsy Multiple axial lesions seen on CT scan

Palpable hepatomegaly and lymphadenopathy, confirmed by CT scan

Lower extremity edema, an episode of anasarca

Primary adrenal insufficiency, gynecomastia,

subclinical primary hypothyroidism

Thrombocytosis

The patient fulfilled the mandatory criteria of peripheral neuropathy and monoclonal plasma cell disorder, as well as two major criteria: osteosclerotic bone lesions, CD; and some of the minor criteria: endocrinopathy, organomegaly, thrombocytosis and extravascular volume overload (Table 2).

\section{Treatment}

Glucocorticoid and mineralocorticoid substitution therapy was prescribed (hydrocortisone $20 \mathrm{mg} /$ day, fludrocortisone $50 \mu \mathrm{g} /$ day) and clinical improvement ensued: psychological well-being, improved muscular strength and capacity to walk after physiotherapy. Normalization of potassium level was also observed, without resin therapy. Due to peripheral edema and mild hypertension, fludrocortisone was subsequently reduced to $50 \mu \mathrm{g}$ every other day, hydrocortisone was replaced by prednisolone $(5 \mathrm{mg})$ and calcium channel blocker wa started (amlodipine $10 \mathrm{mg}$ ). After glucocorticoid replacement therapy, thyroid function was reevaluated, confirming primary subclinical hypothyroidism and levothyroxine was started.

The patient was also treated with analgesics, including morphine, due to the severe pain in the lower limbs, and started physiotherapy with improvement of the gait and hand movements.

After the results of bone marrow biopsy, an autologous hematopoietic cell transplantation (HCT) was planned and cyclophosphamide induction was started in April 2016.

\section{Outcome and follow-up}

Thepatienthad severalcommunity-acquired and nosocomial pneumonias, as well as an episode of pulmonary edema. Despite the fact that the patient started systemic therapy with cyclophosphamide and showed apparent neurologic improvement, he suffered two ischemic strokes (May and August 2016), which resulted in aphasia and hemiparesis. Cerebral angiography revealed vascular lesions compatible with vasculitis and stenosis of right cerebral anterior artery and left middle cerebral artery. These complications and clinical deterioration contraindicated the HCT and the patient deceased 14 months after the diagnosis due to recurrent pulmonary infections.

\section{Discussion}

The young age at presentation, multiplicity of manifestations, the laboratory abnormalities along with the absence of serum monoclonal protein made the diagnosis challenging. We report this case to highlight the importance of consideration of POEMS syndrome in the differential diagnosis of peripheral neuropathy in association with endocrine abnormalities even in young patients. The mandibular mass was presumed to be related to osteomyelitis; however, $S$. mutans and $S$. sanguinis are common inhabitants of the oral cavity and histopathology was nondiagnostic. It could possibly be one of the manifestations of POEMS syndrome.

The pathophysiology is not well understood, though at least in some part it seems to be related to cytokines. 
Endocrinopathies are a frequent feature of POEMS syndrome, but the cause is unknown, with normal structural appearance of endocrine glands at autopsy and no circulating antibodies being found. Therefore, it seems likely that the endocrine abnormalities reflect a functional rather than structural disturbance of gland function (5).

Numerous endocrine abnormalities have been described in POEMS syndrome, and based on the largest published series from Mayo Clinic, which included 64 patients with endocrine data, hypogonadism (primary or secondary) is the most common finding (79\% of tested men) (5). Other frequent endocrinopathies include hypothyroidism (58\% of tested patients) and abnormalities in glucose metabolism (fasting glucose impairment or diabetes, $48 \%$ ); however, it is important to emphasize that these disorders are insufficient to satisfy minor criteria for POEMS syndrome, due to their high prevalence in general population (5). Hypocalcemia is less frequently reported ( $27 \%$ of tested patients in Mayo series) and few cases of adrenal insufficiency have been described (in Mayo series six of nine patients tested) (5).

In another report from University College London Hospitals, with available endocrine data for 59 patients, the most common abnormality was hypogonadism in $68 \%$, central or primary, the latter being more common and developing in the majority of patients after receiving melphalan treatment (6). The treatment with testosterone was associated with increased risk of polycythaemia. Other endocrine abnormalities included hyperprolactinemia found in $56 \%$ of patients, hypothyroidism in $54 \%$ - in all, but one primary, abnormal glucose metabolism in $24 \%$, high IGF-1 in $14.8 \%$ - with growth hormone suppression during $75 \mathrm{~g}$ oral glucose tolerance test, and adrenal insufficiency in $17 \%$ of patients, half of these with primary adrenal insufficiency, one central, and the rest of these patients with secondary cortisol deficiency due to long-term steroid treatment (6). During the follow-up spontaneous resolution of some of the endocrinopathies was observed; however, none of the patients recovered from adrenal insufficiency (6). None of the patients presented hypo- or hyperparathyroidism (6).

In this case, the patient presented multiple endocrine abnormalities: Addison's disease, hypothyroidism and hypocalcemia with elevated PTH, suggestive of secondary hyperparathyroidism, which could be due to vitamin D deficiency; however, PTH elevation was also present, while 25-hydroxy vit. D concentration was normal (Fig. $3)$. The last finding is consistent with the results of the few patients from Mayo Clinic who had hypocalcemia and who had parathyroid function tested (5).

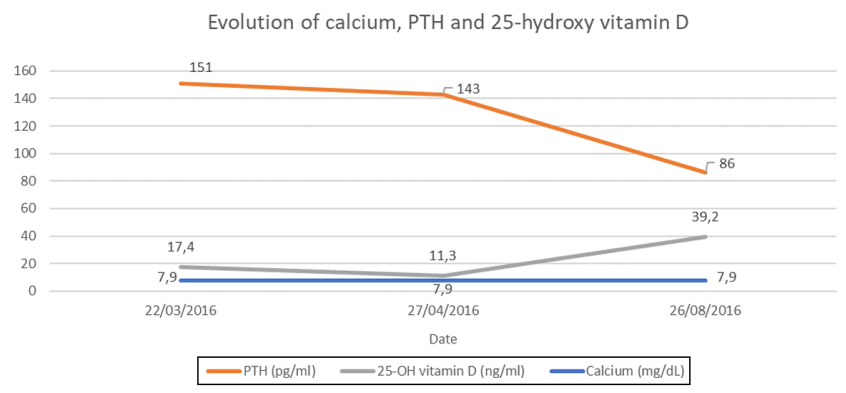

Figure 3

Evolution of calcium, PTH and 25-hydroxy vitamin D.

Symptoms suggestive of primary adrenal insufficiency such as skin hyperpigmentation and postural hypotension were difficult to elicit in this patient: the patient is of African origin and did not notice deepening of his skin color or palmar lines and he was unable to maintain orthostatism. The elevation of potassium level in association with normal renal function, normal renin level and low normal aldosterone were surprising and the short Synacthen test was performed, which revealed lack of significant increment of cortisol as well as aldosterone concentration. The DHEAS was not measured, but the Synacthen test confirmed adrenal insufficiency, and after gluco- and mineralocorticoid replacement therapy, the potassium level returned to normal level, without resin therapy. The lack of elevation of renin despite the primary adrenal insufficiency is unclear; however, it could be potentially provoked by volume expansion associated with POEMS syndrome. POEMS syndrome can also be associated with hyporeninemic hypoaldosteronism, which is usually accompanied by a decline in glomerular filtration rate, metabolic acidosis classified as renal tubular acidosis type IV or proteinuria, which were absent in this case $(7,8)$. We found only one case report of hyporeninemic hypoaldosteronism in POEMS syndrome with hyperkalemia alone (7). The other causes of hyperkalemia such as auto-immune diseases associated with interstitial nephritis were improbable as the autoimmune study was negative, and no anomalies in urinary sediment were found. The patient had no previous history of medication that could potentially impair renal potassium excretion. The other cause that can contribute to the elevation of serum potassium is thrombocytosis, as this electrolyte moves out of platelets after clotting has occurred, provoking pseudohyperkalemia. During the hospitalization low potassium diet was started, however, without impact upon the electrolytes.

The patient presented subclinical hypothyroidism, which persisted after glucocorticoid replacement 
therapy. The levothyroxine therapy should be initiated after starting treatment of adrenal insufficiency. In hypothyroidism prolonged half-life of cortisol is observed and the addition of thyroid hormone replacement therapy increases cortisol metabolism, which is why the thyroxin therapy can precipitate acute adrenal crisis if the gluco- and mineralocorticoid requirements are not met (9). Furthermore, the elevation of TSH may be a feature of adrenal insufficiency. The elevation of TSH can also be observed during recovery from severe nonthyroidal illness; however, in this case, the repeated thyroid function testing confirmed the primary subclinical hypothyroidism.

The patient did not complain of erectile dysfunction or loss of body hair, but presented gynecomastia. The laboratory work-up revealed total testosterone around the lower limit of the normal range and low free testosterone, measured by radioimmunoassay, with elevation of gonadotrophic hormones. The radioimmunoassay is inaccurate in determining the free testosterone concentration, and it should be performed directly by equilibrium dialysis assay or calculated on the basis of the total testosterone, albumin and SHBG concentration. Unfortunately, the SHBG testing was not performed and the laboratory of the hospital did not perform equilibrium dialysis assay. The primary hypogonadism was probable, but not confirmed, given that the total testosterone was still in the normal range and SHBG was not measured. Nevertheless, at the time of diagnosis, it did not seem that confirmation of primary hypogonadism would alter the clinical management of this patient.

The neuropathy is the dominant characteristic of POEMS syndrome and it is peripheral, ascending, symmetric and affecting both sensation and motor function. In about $10-15 \%$ of patients pain may be a dominant feature. On neurologic evaluation patients are areflexic and typically have a steppage gait and a positive Romberg sign (10). Nerve conduction studies show slowing of nerve conduction, prolonged distal latencies and severe attenuation of compound muscle action potentials (11). In this case the disorder was already advanced and the nerve action potentials were unrecordable, with the exception of the motor potentials in the upper limbs, which were consistent with characteristics of POEMS neuropathy. The sural nerve biopsy usually shows axonal degeneration and demyelination, as seen in this patient (3).

In this case, although serum immunofixation was negative for monoclonal protein, bone marrow biopsy confirmed POEMS syndrome. The syndrome is considered 'low tumor burden disease' and the monoclonal protein in this condition is usually less than $2 \mathrm{~g} / \mathrm{dL}$, in $15 \%$ of cases not being found by immunofixation (3).

Castelman's disease and POEMS syndrome are frequently associated, overproduction of cytokines such as interleukine- 6 being probably implicated in their pathogenesis. Although human herpes virus 8 (HHV-8) is also involved in pathogenesis of CD mainly, but not only, in HIV-positive patients (12), HIV serology and immunohistochemistry of the lymph node for HHV-8 were negative.

There is no standard therapy; however, patients with disseminated bone marrow involvement are treated with chemotherapy with or without HCT (13). In this patient, despite the young age, the disease was already advanced and resulted in severe complications, which led to abandon the intention of HCT. He suffered recurrent ischemic strokes, which are rarely described in patients with POEMS syndrome. In 28 reported cases with POEMS syndrome and ischemic stroke, $71.5 \%$ were found to have multiple ischemic lesions and $48 \%$ had more than one cerebral artery involved. Ischemic stroke in patients with POEMS syndrome is likely to be a poor outcome predictor (14). In fact, the patient deceased 14 months after the diagnosis, while the median survival of patients with POEMS syndrome is about 165 months (3).

\section{Declaration of interest}

The authors declare that there is no conflict of interest that could be perceived as prejudicing the impartiality of the research reported.

\section{Funding}

This research did not receive any specific grant from any funding agency in the public, commercial or not-for-profit sector.

\section{Patient consent}

The patient is deceased and the authors sought the consent from his relatives. The patient was from Guinea Bissau, and we reached the brother of the patient, who gave the consent.

\section{Author contribution statement}

$J \mathrm{P}$ wrote this case report during her trainee program in the Endocrinology Department in 'Centro Hospitalar Universitário Lisboa Central'. J E was involved in clinical disease diagnosis. J P and T S were the patient's Endocrinology attending physicians. S M was the patient's Internal Medicine attending physician. E S was the clinical head of the Internal Medicine Department. A B was the patient's Hematology attending physician. A A was the head of the Endocrinology Department and supervised the management and care of patient. 


\section{References}

1 Dispenzieri A. POEMS syndrome: 2014 update on diagnosis, riskstratification, and management. American Journal of Hematology 2014 89 214-223. (https://doi.org/10.1002/ajh.23644)

2 Gherardi RK, Bélec L, Soubrier M, Malapert D, Zuber M, Viard JP, Intrator L, Degos JD \& Authier FJ. Overproduction of proinflammatory cytokines imbalanced by their antagonists in POEMS syndrome. Blood 199687 1458-1465.

3 Dispenzieri A, Kyle RA, Lacy MQ, Rajkumar SV, Therneau TM, Larson DR, Greipp PR, Witzig TE, Basu R, Suarez GA, et al. POEMS syndrome definitions and long-term outcome. Blood 2003101 2496-2506. (https://doi.org/10.1182/blood-2002-07-2299)

4 Dispenzieri A. POEMS syndrome. Blood Reviews 200721 285-299. (https://doi.org/10.1016/j.blre.2007.07.004)

5 Gandhi GY, Basu R, Dispenzieri A, Basu A, Montori VM \& Brennan MD. Endocrinopathy in POEMS syndrome: the Mayo Clinic experience. Mayo Clinic Proceedings 200782 836-842. (https://doi. org/10.4065/82.7.836)

6 Caimari F, Keddie S, Lunn MP, D’Sa S \& Baldeweg SE. Prevalence and course of endocrinopathy in POEMS syndrome. Journal of Clinical Endocrinology and Metabolism 2018104 2140-2146. (https://doi. org/10.1210/jc.2018-01516)

7 Rey JP, Modesto A, Orfila C, Cozette P, Donnadieu P, Lauze I, Héran B, Bertrand JL, Camo J, Négre C, et al. Manifestation rénales du syndrome POEMS. Nephrologie 199415 335-338.
8 Nakamoto Y, Imai H, Yasuda T, Wakui H \& Miura AB. A spectrum of clinicopathological features of nephropathy associated with POEMS syndrome. Nephrology, Dialysis, Transplantation 199914 2370-2378. (https://doi.org/10.1093/ndt/14.10.2370)

9 Iranmanesh A, Lizarralde G, Johnson ML \& Veldhuis JD. Dynamics of 24-hour endogenous cortisol secretion and clearance in primary hypothyroidism assessed before and after partial thyroid hormone replacement. Journal of Clinical Endocrinology and Metabolism 199070 155-161. (https://doi.org/10.1210/jcem-70-1-155)

10 Dispenzieri A. POEMS syndrome: 2017 update on diagnosis, risk stratification, and management. American Journal of Hematology 2017 92 814-829. (https://doi.org/10.1002/ajh.24802)

11 Min JH, Hong YH \& Lee KW. Electrophysiological features of patients with POEMS syndrome. Clinical Neurophysiology 2005116 965-968. (https://doi.org/10.1016/j.clinph.2004.11.011)

12 Fajgenbaum DC, van Rhee F \& Nabel CS. HHV-8-negative, idiopathic multicentric Castleman disease: novel insights into biology, pathogenesis, and therapy. Blood 2014123 2924-2933. (https://doi. org/10.1182/blood-2013-12-545087)

13 Dispenzieri A. How I treat POEMS syndrome. Blood 2012119 5650-5658. (https://doi.org/10.1182/blood-2012-03-378992)

14 Fu FW, Rao J, Zheng YY, Wang HL, Yang JG \& Zheng GQ. Ischemic stroke in patients with POEMS syndrome: a case report and comprehensive analysis of literature. Oncotarget 20178 89406-89424. (https://doi.org/10.18632/ oncotarget.20131)

Received in final form 24 June 2019

Accepted 16 July 2019 